\title{
Interface core-level shifts as a probe of embedded thin-film quality
}

\author{
Weine Olovsson, Erik Holmstroem, Tobias Marten, \\ Igor Abrikosov and Anders M N Niklasson
}

\section{Linköping University Post Print}

N.B.: When citing this work, cite the original article.

Original Publication:

Weine Olovsson, Erik Holmstroem, Tobias Marten, Igor Abrikosov and Anders M N Niklasson, Interface core-level shifts as a probe of embedded thin-film quality, 2011, Physical Review B. Condensed Matter and Materials Physics, (84), 8, 085431.

http://dx.doi.org/10.1103/PhysRevB.84.085431

Copyright: American Physical Society http://www.aps.org/

Postprint available at: Linköping University Electronic Press

http://urn.kb.se/resolve?urn=urn:nbn:se:liu:diva-70748 


\title{
Interface core-level shifts as a probe of embedded thin-film quality
}

\author{
Weine Olovsson, ${ }^{1}$ Erik Holmström, ${ }^{2}$ Tobias Marten, ${ }^{1}$ Igor A. Abrikosov, ${ }^{1}$ and Anders M. N. Niklasson ${ }^{3}$ \\ ${ }^{1}$ Department of Physics, Chemistry and Biology (IFM), Linköping University, SE-581 83 Linköping, Sweden \\ ${ }^{2}$ Instituto de Ciencias Físicas y Matemáticas, Universidad Austral de Chile, Valdivia, Chile \\ ${ }^{3}$ Theoretical Division, Los Alamos National Laboratory, Los Alamos, New Mexico 87545, USA \\ (Received 14 December 2010; revised manuscript received 11 July 2011; published 26 August 2011)
}

\begin{abstract}
We use first-principles calculations of layer-resolved core-level binding energy shifts (CLSs) within density functional theory as a way to characterize the interface quality and thickness in embedded thin-film nanomaterials. A closer study of interfaces is motivated as properties specific to nanostructures can be related directly to the interface environment or indirectly as interference effects due to quantum confinement. From an analysis based on the $\mathrm{Cu} 2 p_{3 / 2} \mathrm{CLS}$ for $\mathrm{Cu}$ embedded in $\mathrm{Ni}$ and $\mathrm{Co}$ fcc (100) and Fe bcc (100), with the interfaces represented by intermixing profiles controlled by a single parameter, we evaluate layer-resolved shifts as a probe of the thin-film quality. The core-level shifts in the corresponding disordered alloys, as well as local environment effects, are studied for comparison. We also discuss the possibility of detecting interface states by means of core-level shift measurements.
\end{abstract}

DOI: 10.1103/PhysRevB.84.085431

PACS number(s): 71.15.-m, 79.60.Jv, 79.60.Ht

\section{INTRODUCTION}

The existence of interfaces and finite-size effects due to quantum interference in nanomaterials gives rise to the possibility of designing new properties, magnetic, optical, and mechanical, by changing interface compositions and by controlling interface quality and geometry. In order to better understand how the interfaces can be used to control the properties of materials at the nanoscale it is important to investigate methods that can be used to analyze the interfaces in great detail. This is particularly important in the case of thin-film nanomaterials where the thickness of the film directly determines how the material properties are governed by interface effects. Since core-electron binding energies are element specific but, at the same time, highly sensitive to the chemical environment of the atom, the so-called core-level binding energy shift (CLS) is a very promising candidate for such structural characterizations of materials. ${ }^{1}$ However, CLS measurements provide only statistical information, i.e., structural information on the atomic level cannot be determined from the spectrum alone. In fact, the most rigorous way to characterize a structure from experimental CLS results is to calculate the CLS of a set of model structures from first principles and compare the results to the measurements. The model structure that describes the experiment best may then be considered the archetypal structure.

Recently, we have used theoretical layer-resolved CLS of model structures ${ }^{2,3}$ together with measurements from nondestructive high-kinetic-energy photoelectron spectroscopy to analyze the interface quality of $\mathrm{Cu} / \mathrm{Ni}$ multilayered systems. ${ }^{4}$ In the present work we evaluate first-principles calculations of layer-resolved core-level shifts as a tool for characterizing interface qualities and thickness in a broader range of embedded thin-film nanomaterials. The investigation is done by a systematic comparison of layer-resolved shifts at the interfaces between metals $A$ and $B$ for different interface quality, or intermixing of atoms, at the embedded $B / A / B$ interfaces. We describe the composition profiles by using a single parameter for the intermixing. ${ }^{5}$ In combination with Green's function methods based on the coherent potential approximation (CPA) ${ }^{6-10}$ all within the framework of density functional theory (DFT), ${ }^{11,12}$ this allows for a very efficient computation. To determine the CLSs the well-established complete screening picture for metallic systems is employed, including both the initial and final-state effects of the photoemission process. ${ }^{3,13}$ Some recent applications include the shift as a function of the composition in binary disordered alloys ${ }^{14}$ and the broadening of the spectral core line in disordered alloys due to different local environment of the atoms. ${ }^{15}$ In a follow-up study, segregation effects as the structure evolves with temperature were stressed. ${ }^{16}$ The results for embedded $\mathrm{Cu} / \mathrm{Ni}$ systems have also been discussed in the reviews. ${ }^{17,18}$ Here, we compare shifts as a function of composition over the corresponding disordered alloys and estimate local environment effects for equiatomic alloys (disorder broadening).

For this study we consider the layer resolved $\mathrm{Cu} 2 p_{3 / 2}$ interface CLS in different thin-film embedded nanosystems, namely $\mathrm{Ni} / \mathrm{Cu}_{N} / \mathrm{Ni}$ fcc (100), $\mathrm{Co} / \mathrm{Cu}_{N} / \mathrm{Co} \mathrm{fcc}$ (100), and $\mathrm{Fe} / \mathrm{Cu}_{N} / \mathrm{Fe}$ bcc (100) sandwiches, for up to $N=10$ layers of spacer thickness and three different composition profiles ranging from ideal to more intermixed interfaces. Our calculated broadenings and spectra can be directly compared to high-resolution photoemission spectra.

The article is outlined as follows: First, we discuss the computational details, namely how to calculate core-level shifts within the complete screening picture and the construction of the interface mixing profile. Thereafter we describe the results obtained for the different alloys and thin-film nanosystems. In addition, interface states in the $\mathrm{Cu}-\mathrm{Fe}$ systems are investigated. At the end we discuss some possibilities to explore the distribution of shifts to estimate the quality of an intermixed binary alloy interface. In the appendix we tabulate the layer-resolved core-level shifts for the different material combinations.

\section{CALCULATiONAL DETAILS}

\section{A. Computational method}

The ab initio analysis of the electronic structure of the thinfilm nanomaterials and disordered alloys has been performed within DFT. ${ }^{11,12}$ The computational methods are based on the 
scalar relativistic spin-polarized linearized-muffin-tin-orbitalmethod and KKR-ASA ${ }^{8-10,19-23}$ formulated as a Green's function technique. The advantages of the Green's function formulation of the Kohn-Sham equations are the flexibility to introduce a variety of different boundary conditions and to describe substitutional disorder within the coherent potential approximation (CPA). In the case of the layered nanomaterials, the method allows the construction of boundary conditions that correspond to interfaces between two semi-infinite bulk crystals without the complication of artificial periodic boundary conditions. Only the general ideas will be outlined here and for a complete description of the method we refer to Refs. 8-10 and 23-26.

First, Green's functions of the two crystals that will make up the interface are obtained from normal three-dimensional KKR-ASA band calculations. These Green's functions are then Löwdin-downfolded to form boundary conditions for the interface calculation. The two-interface Green's functions are then combined to form Green's function matrices of an ideal interface where the charge density has not yet been relaxed. The electronic structure problem of the interface region where the charge density differs from the ideal bulk density is then solved self-consistently in a Dyson equation for the atomic layers closest to the interface.

The interface roughness between the interfaces is described by a layer-resolved binary alloy $A_{1-c}(n) B_{c}(n)$ with a concentration $c$ that depends on the atomic monolayer $n$. The alloys are described in the framework of single-site CPA and the methods are well adapted to close-packed metallic systems since the atomic sphere approximation is used.

The thin-film nanosystems consist of an $N=1-10$ monolayer (MLs) thick slab of material $A_{N}$, embedded between two semi-infinite crystals of material $B$, which are put together to form a $B / A_{N} / B$ sandwich. Far away from the interface, the electronic structure equals that of the corresponding bulk $B$ metal. The geometry at the interface has not been relaxed and the same lattice structure is used for the entire sandwich.

In order to estimate local environment effects, supercell calculations were performed within the order- $N$ locally selfconsistent Green's function (LSGF) method. ${ }^{27,28}$ The multiple scattering problem is solved for each and every atom, considering a local interaction zone centered on the atom, here chosen as two coordination shells, embedded into an effective medium determined by CPA.

\section{B. Core-level shifts}

We obtain the core-level binding energy shifts within the complete screening picture, ${ }^{1}$ including both initial and finalstate effects simultaneously in the computation scheme. The initial state corresponds to the unperturbed system in its ground state before the photoemission. The difference between coreelectron eigenenergies in the ground state (referenced to the Fermi level) is usually referred to as the initial-state core-level shift (is-CLS) in the context of DFT calculations. The final state can be represented by introducing a single core-ionized atom, with the core-hole fully screened by the valence charge, valid for metallic systems. In our calculations this is modeled by promoting the core-electron from the ionized atom into the valence band. The reference binding energy for the CLS in an atom is, in principle, arbitrary but, for practical and comparison reasons, usually chosen as the corresponding pure bulk metal, in this work $\mathrm{Cu}$.

The complete screening picture has been successfully employed in a number of studies; in the beginning it was applied to the shift between the free atom and metal, ${ }^{1}$ and it has been used for both bulk ${ }^{3,14,29}$ and surface core-level shifts. ${ }^{30,31}$ As previously mentioned it was recently used to estimate the broadening of the spectral core line in substitutional random alloys, i.e., disorder broadening, with good agreement compared to experimental values. ${ }^{15}$ For an overview of different applications and studies conducted by some of the authors, including core-level shifts and other spectral properties, see, e.g., Refs. 17 and 32.

\section{Single-site approximation}

To calculate the shifts in the disordered alloy systems using the CPA formalism, we employ the generalized thermodynamic chemical potential (GTCP) of the core-ionized atoms ${ }^{3}$

$$
\mu=\left.\frac{\partial E_{\mathrm{tot}}}{\partial c}\right|_{c \rightarrow 0} .
$$

Here $E_{\text {tot }}$ is the total energy of a system in which a specific core-electron of an atom has been ionized, with a total concentration of $c$ ionized atoms. The limit $c \rightarrow 0$ is taken from extrapolation of $E_{\text {tot }}$ calculated at different concentrations $c$. Note that this is a proper way of obtaining chemical potentials inside the formalism of the CPA single-site approximation. ${ }^{33}$

The method is easy to generalize to different kinds of binding energy shifts. The layer resolved shifts in the thin embedded films can be calculated by considering the GTCPs in Eq. (1) separately for each particular atomic monolayer $n$ in the thin-film nanosystem, $\mu(n)$. The interface core-level shift ${ }^{2}$ studied in the present work is given by the difference,

$$
E_{\mathrm{ICLS}}(n)=\mu_{\mathrm{IF}}(n)-\mu_{\text {bulk }},
$$

where the chemical potential $\mu_{\mathrm{IF}}(n)$ corresponds to the interface layer and $\mu_{\text {bulk }}$ to the pure bulk metal. In practice, this means that in order to obtain the GTCP of all layers in a system, one separate self-consistent calculation must be performed for each layer.

\section{Supercell technique}

The above formulation of the complete screening picture is applied to the case of single-site approximation, i.e., local environment effects are ignored. For an alloy simulated by means of a supercell technique, the generalized thermodynamic chemical potentials become site dependent, since we can have different local environments around every atom site $i$. Using the LSGF method, for an $N$-atom supercell the GTCP at each site can be calculated as $[c=1 / N$ in Eq. (1)]

$$
\mu_{i}=N\left(E_{i}^{*}-E^{\mathrm{gs}}\right),
$$

where $E_{i}^{*}$ denotes the total energy for the $A B$ alloy or pure $A(B)$ metal with core-ionized atom $\left(A^{*}\right.$ or $\left.B^{*}\right)$ situated at site $i$. $E^{\mathrm{gs}}$ corresponds to the ground state, that is, the unperturbed system without any core-ionized atom. Note that in a supercell method that instead uses an extensive formulation of the total energy (an approach with $E_{\text {tot }}$ summed over the atoms), Eq. (1) 
TABLE I. Lattice parameters for the fcc $\mathrm{CuNi}, \mathrm{CuCo}$ alloys, bcc $\mathrm{CuFe}$ alloy, and fcc $\mathrm{Cu}$ that were used in the different calculations.

\begin{tabular}{lcccc}
\hline \hline & $\mathrm{CuNi}(\AA)$ & $\mathrm{CuCo}(\AA)$ & $\mathrm{CuFe}(\AA)$ & $\mathrm{Cu}(\AA)$ \\
\hline Supercell & 3.55 & 3.55 & 2.85 & 3.61 \\
Bulk \& interf. & 3.52 & 3.49 & 2.83 & 3.57 \\
\hline \hline
\end{tabular}

reduces to a difference between total energies for the ground state and the perturbed, core-ionized, system.

By using LSGF it is possible to calculate both the average CLS and the dependence of this shift on the next nearestneighbor environment of an atomic site. Comparing the GTCPs at each site gives the site specific core-level shift. The average CLS is readily obtained from the values at individual sites. As a measure of the variation of CLS, we use the Gaussian full width at half maximum (FWHM), $\Gamma=2 \sigma \sqrt{2 \ln 2}$, where $\sigma$ is the standard deviation of the distribution.

The disordered equiatomic $A B$ alloys are modeled by a special quasirandom structure (SQS) ${ }^{34,35}$ containing 256 atoms. Self-consistent solutions are obtained using the local density approximation (LDA) with a last additional iteration where the generalized gradient approximation (GGA) is applied, both functionals are parametrized according to Ref. 36. Further, a $s p d f$ basis set was used. Other details of the calculations follow the scheme outlined in Ref. 15. This differs from the case of the disordered alloys and layered systems, where LDA was employed together with a $s p d$ basis set, as in Ref. 2. Lattice parameters are obtained by minimization of the total energy for the unperturbed systems, and thereafter the same volume is used in calculations of the total energy in its perturbed state. Volumes are explicitly given in Table I.

\section{Interface mixing}

Here the interface roughness for the thin-film nanosystem is described by a partial intermixing of the two constituent interface materials. Consider a single interface between two metals, $A / B$. The concentration profile around the interface due to intermixing can be modeled by a layer-resolved binary alloy composition profile $A_{1-c}(n) B_{c}(n)$. The distribution determining the concentration profile is given by a general normal cumulative distribution function

$$
\Lambda\left[X, \Gamma_{C}\right]=\frac{1}{\Gamma_{C} \sqrt{2 \pi}} \int_{-\infty}^{X} e^{\frac{x^{2}}{2 \Gamma_{C}^{2}}} d x
$$

centered around the interface. In Eq. (4), $X$ is the distance from the interface (centered in the middle between the atomic layers on each side of the interface) and $\Gamma_{C}$ is the standard deviation that determines the width of the interface mixing. Note that this model is a simplification, since the inherent surface diffusion of the elements strongly depends on the material combination.

The concentration profile $C\left(X, \Gamma_{C}\right)$ of the trilayer interface described by the layer resolved binary alloy $A_{1-c}(n) B_{c}(n)$ is obtained as a sum of the layers in the sample, ${ }^{5}$ and the expression for the trilayer concentration is

$$
C\left(X, \Gamma_{C}\right)=\Lambda_{-1}\left(-X, \Gamma_{C}\right)+\Lambda_{1}\left(X, \Gamma_{C}\right) .
$$

Here $\Lambda_{i}$ is centered at interface $i$, see Fig. 1. Setting, for example, interface $i=1$ as $X=0$ and counting layers from

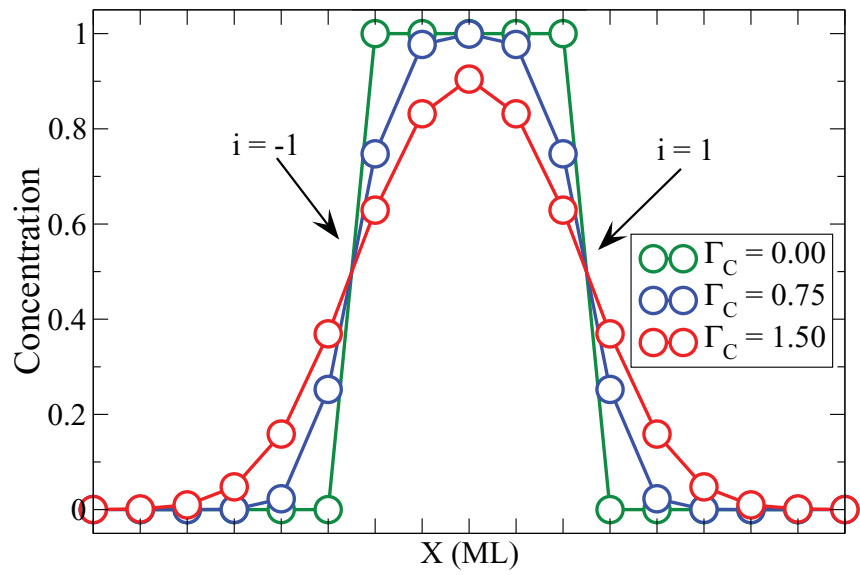

FIG. 1. (Color online) Concentration profile for a $B / A_{6} / B$ system with interface alloying parameters $\Gamma_{C}=1.50,0.75$, and 0.00 .

this interface, we obtain the layer concentration at layer $n$ as $C\left(n-0.5, \Gamma_{C}\right)$. Once the concentration profile is obtained, the interface core-level shifts can be calculated directly using the method that was outlined above. This interface modeling technique has been applied recently to study interface quality effects in a variety of multilayer systems. ${ }^{4,5,37-42}$ Interfaces between two metals may also intermix as plateaus if the two metal segregate. The interface stability may be estimated from the rescaled formation energy and depend on crystal structure, interface direction, and the thickness of the intermixed region. ${ }^{43}$

Figure 1 illustrates the layer-resolved concentration profile of the $B / A_{6} / B$ interface, described by the binary alloy $A_{1-c}(n) B_{c}(n)$, for three different values of the mixing parameter $\Gamma_{C}$.

\section{Simulation of spectra}

The layer-dependent ICLS can be calculated by means of Eq. (2), with a fixed concentration profile $C(n)$ described by the parameter $\Gamma_{C}$. In order to estimate the photoelectron intensity as function of energy, $E_{\mathrm{ICLS}}(n)$ must be averaged over all layers. One straightforward way is to apply a Gaussian broadening and add the different contributions

$$
I(E)=\frac{1}{N} \sum_{n} g\left(E_{\mathrm{ICLS}}(n), \sigma\right) C(n),
$$

where $g(E, \sigma)$ is a normalized Gaussian function with standard deviation $\sigma$ centered at $E . N$ is the total number of layers of the studied atom before intermixing. In the calculations we have used $\sigma=0.1 \mathrm{eV}$.

\section{RESULTS}

\section{A. Disordered bulk alloys}

In the Figs. 2-4 we demonstrate the $\mathrm{Cu} 2 p_{3 / 2}$ core-level shift as a function of composition and volume in fcc $\mathrm{Cu}_{x} \mathrm{Ni}_{1-x}$, fcc $\mathrm{Cu}_{x} \mathrm{Co}_{1-x}$, and bcc $\mathrm{Cu}_{x} \mathrm{Fe}_{1-x}$ substitutional random alloys, calculated by means of the single-site approximation. The respective theoretical equilibrium volumes are denoted by + in the figures. For clarity, contour lines are plotted in steps of $0.1 \mathrm{eV}$. Starting with the CuNi alloys in Fig. 2, at small $x$ 


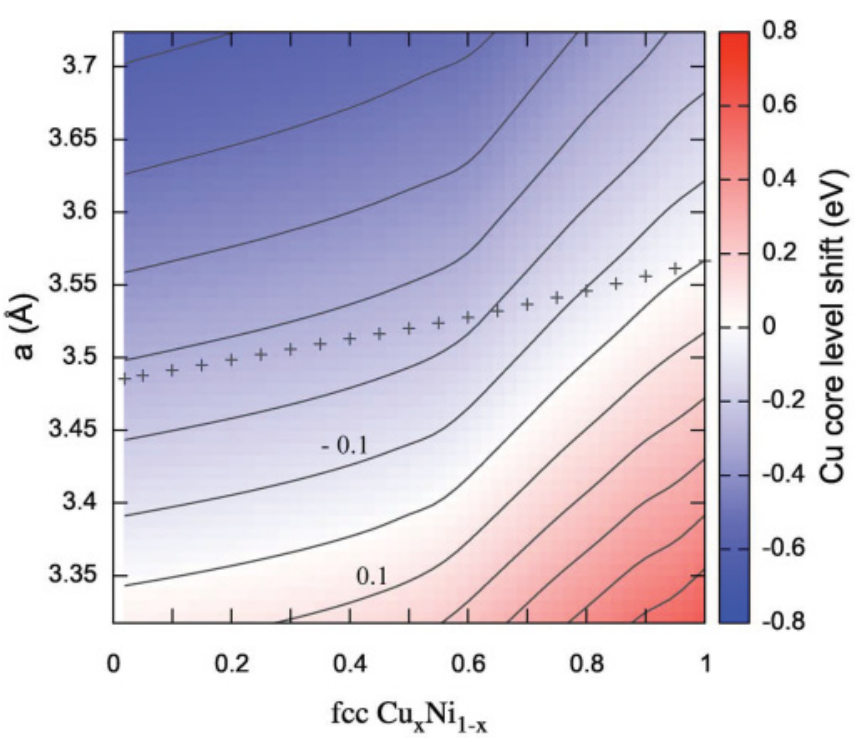

FIG. 2. (Color online) $\mathrm{Cu} 2 p_{3 / 2} \mathrm{CLS}(\mathrm{eV})$ as a function of the lattice parameter $a(\AA)$ and $\mathrm{Cu}$ atom concentration $x$ in the fcc $\mathrm{Cu}_{x} \mathrm{Ni}_{1-x}$ alloys. Contour lines are in steps of $0.1 \mathrm{eV}$ and theoretical equilibrium volumes are marked + .

the CLS is $-0.27 \mathrm{eV}$ and changing slowly until about $x=0.6$ where it changes more rapidly and increases to zero at $x=1$. This follows our previous results in Ref. 14. In the earlier work it was found that the increasing magnetic moment at the $\mathrm{Ni}$ atoms for $x \leqslant 0.5$ produces a smaller shift at $\mathrm{Cu}$ as compared to the nonmagnetic alloy. Experimental measurements of the CLSs over the CuNi alloys can be found in Refs. 44 and 45, with comparison to theory in Ref. 14.

The $\mathrm{Cu}$ core-level shift at equilibrium volumes in random fcc CuCo alloys, Fig. 3, varies less compared with $\mathrm{CuNi}$. A small negative shift is noted over the compositions $x \geqslant 0.25$, while a positive shift of $0.05 \mathrm{eV}$ is obtained at the dilute limit

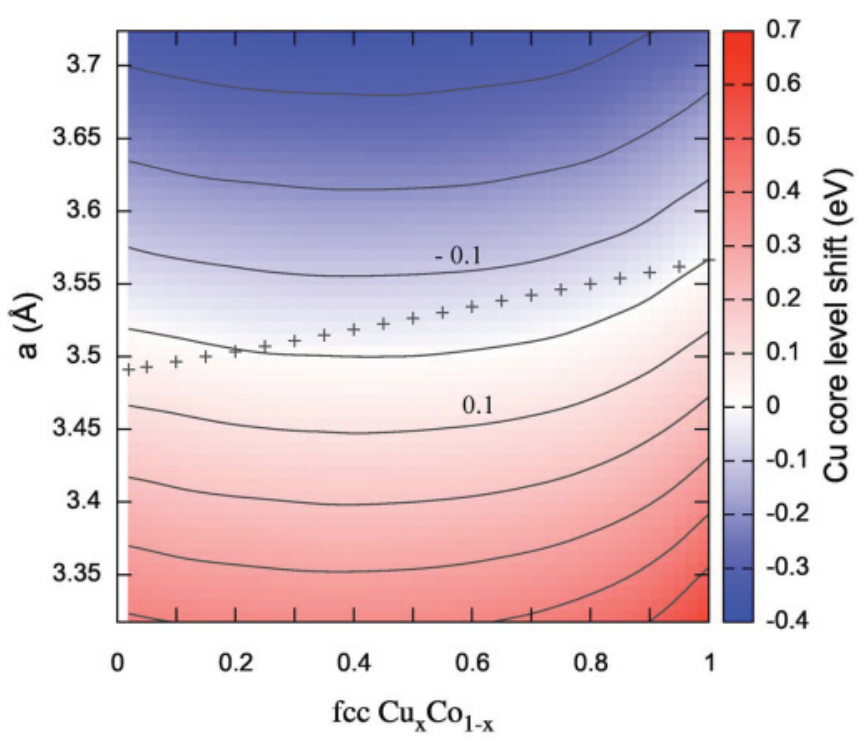

FIG. 3. (Color online) $\mathrm{Cu} 2 p_{3 / 2} \mathrm{CLS}$ as a function of the lattice parameter $a(\AA)$ and $\mathrm{Cu}$ atom concentration $x$ in the fcc $\mathrm{Cu}_{x} \mathrm{Co}_{1-x}$ alloys. Notations are the same as described in the caption to Fig. 2.

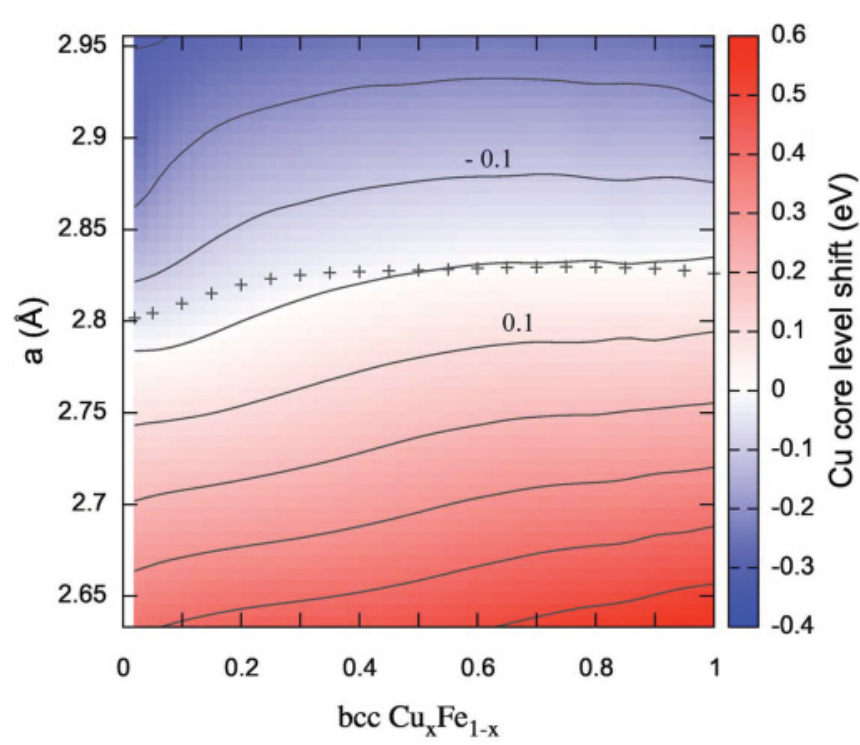

FIG. 4. (Color online) $\mathrm{Cu} 2 p_{3 / 2} \mathrm{CLS}$ as a function of the lattice parameter $a(\AA)$ and $\mathrm{Cu}$ atom concentration $x$ in the bcc $\mathrm{Cu}_{x} \mathrm{Fe}_{1-x}$ alloys. Notations are the same as described in the caption Fig. 2.

$(x \rightarrow 0)$. In contrast with $\mathrm{CuNi}$, the fcc disordered phase is not energetically stable over the full range of CuCo alloys.

Turning to bcc $\mathrm{CuFe}$, Fig. 4, the situation is somewhat similar to $\mathrm{CuCo}$ with a $\mathrm{Cu}$ CLS close to zero. For the comparison, the reference lattice at $x=1$ is chosen as bcc, giving rise to a small positive shift at equilibrium volume. The largest value over the compositions, $-0.05 \mathrm{eV}$, coincides with the dilute limit $x \rightarrow 0$. In experiment, a disordered fcc (bcc) phase occurs at concentrations dominated by $\mathrm{Cu}(\mathrm{Fe})$, with a mixture of the phases in between. We also performed calculations for fcc $\mathrm{Cu}_{x} \mathrm{Fe}_{1-x}$, finding a similar trend of small $\mathrm{Cu}$ shifts close to zero, with the exception of a positive shift, $0.1 \mathrm{eV}$, for high Fe concentrations. Experimental studies report small shifts, or up to $\sim 0.1 \mathrm{eV}$, at different concentrations over the $\mathrm{CuFe}$ alloys. ${ }^{46,47}$

The above trends of the Cu CLS in the alloys can be described in terms of initial and final state effects contributing to the total shift. First, we consider the shift at the theoretical equilibrium volumes. We find that the initial-state shift in $\mathrm{CuCo}$ and $\mathrm{CuFe}$ is positive and increases with lower $\mathrm{Cu}$ atom concentration. For CuNi the is-CLS is instead smaller and negative over the alloy concentrations. ${ }^{14}$ Previously, it has been closely connected to the changes in the valence $d$-band density of states (DOS) ${ }^{3}$. Here, for the $\mathrm{CuFe}$ and $\mathrm{CuCo}$ alloys, a smaller shift of the $d$-band center away from the Fermi level (positive shift) is noted at the $\mathrm{Cu}$ sites, while the corresponding change is closer to zero for $\mathrm{CuNi}$. In comparison with the is-CLS, the total CLS gives more negative values over the alloy concentrations. The result is a total shift close to zero for $\mathrm{CuFe}$ and $\mathrm{CuCo}$, and a larger negative shift in $\mathrm{CuNi}$ alloys. This tendency can be understood by considering the final-state effect, which is sensitive to differences in the orbital character of the charge screening the core hole. Going from $\mathrm{Fe}$ to $\mathrm{Cu}$ in the periodic table, there is a progressive filling of the $d$ band. In the pure $\mathrm{Cu}$ metal, the screening charge will be mostly of $s p$ type, which will be mixed with $d$ character (more efficient screening) in the alloy systems. Compare, e.g., with the $\mathrm{Ag}$ 


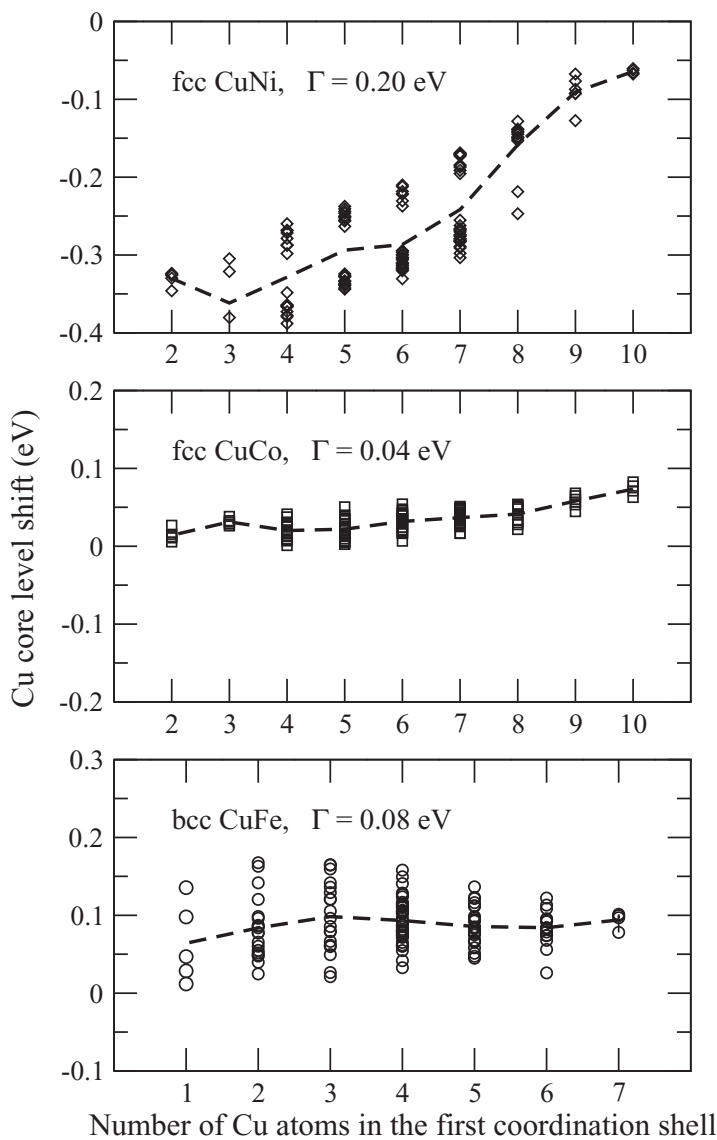

FIG. 5. The $\mathrm{Cu} 2 p_{3 / 2}$ CLS for different local environments in 256 atom SQS supercells as a function of $\mathrm{Cu}$ nearest-neighbor atoms. (From top to bottom) The equiatomic $(x=0.5)$ disordered fcc $\mathrm{CuNi}$, fcc $\mathrm{CuCo}$, and bcc CuFe alloys.

$3 d_{5 / 2}$ initial-state and total CLS in AgPd-alloys. ${ }^{3}$ In CuFe and $\mathrm{CuCo}$, a larger effect is noticed, compared with $\mathrm{CuNi}$. Second, we consider the change over different volumes. For all the studied alloys, the effect of higher pressure gives a tendency toward more positive shifts. Studying the average local DOS at the $\mathrm{Cu}$ atoms, we find that while the valence band $d$-DOS is wider at higher pressures, it narrows closer to the Fermi level for the dilated alloys. This tendency is mirrored by the more deeply confined core levels, resulting in an increasingly positive initial-state CLS with higher pressure.

While the above results from using the single-site approximation give the distribution of average core-level shifts as a function of volume and composition, the specific local environment effects are not included. Therefore, to investigate the dispersion of the shifts, we have calculated the so-called disorder broadening of $\mathrm{Cu} 2 p_{3 / 2}$ at the equiatomic systems (50/50 alloy compositions). In Fig. 5 the shifts due to the different local environments in 256 atom large SQS supercells are displayed as a function of $\mathrm{Cu}$ atoms in the first coordination shell. Going from top to bottom, for fcc $\mathrm{CuNi}$ we can see that some local environments have a spread up to $0.15 \mathrm{eV}$, with a change in the average shift toward larger negative values with decreasing number of neighboring $\mathrm{Cu}$ atoms. The disorder broadening at FWHM is here $\Gamma=0.20 \mathrm{eV}$, with a mean CLS of $-0.26 \mathrm{eV}$. It is interesting to note that there seems to be two groups of shifts for each local environment, which is not displayed in the other investigated alloys. Turning to $\mathrm{CuCo}$, the trend differs with a smaller spread both within and in between the different local environments, $\Gamma=0.04 \mathrm{eV}$, with an average shift of $0.03 \mathrm{eV}$. In the case of $\mathrm{CuFe}$ a larger spread around each local environment can be found, as compared to CuCo. However, in contrast to $\mathrm{CuNi}$, the different local environments do not add up to a total larger broadening. The disorder broadening was calculated to $\Gamma=0.08 \mathrm{eV}$ with an average shift of 0.09 $\mathrm{eV}$. These results indicate that while the disorder broadening of $\mathrm{Cu}$ in $\mathrm{CuNi}$ might be measured experimentally, the other alloys would prove more difficult. One can note that the local environment effects roughly follow the dispersion of the average shifts seen in Figs. 2-4. Numerical differences between the two approaches are due to the computational details and are not crucial, since we are investigating general trends over many systems rather than a single CLS.

It has been shown that the effect of local lattice relaxation can have a strong impact on the shifts. ${ }^{48}$ However, in the present case it is not expected, due to the similar size of the participating atoms. The obtained results demonstrate the overall sensitivity of the CLS on volume and composition, as well as local environment effects, in the respective disordered alloys, providing a useful background for the study of the interface CLS of the layered systems in the next section.

\section{B. Embedded thin films}

In this section we demonstrate first-principles calculations of layer-resolved $\mathrm{Cu} 2 p_{3 / 2}$ interface core-level shifts as a function of interface quality and embedded film thickness, $N=1-10 \mathrm{MLs}$, for fcc (100) $\mathrm{Ni} / \mathrm{Cu}_{N} / \mathrm{Ni}$ and $\mathrm{Co} / \mathrm{Cu}_{N} / \mathrm{Co}$ and bcc (100) $\mathrm{Fe} / \mathrm{Cu}_{N} / \mathrm{Fe}$. The atom concentration profiles that model the different interface qualities are produced by a single parameter, as described in Sec. II C. In the present investigation we chose $\Gamma_{C}=0,0.75$, and 1.5 , giving a perfect sharp interface and two increasingly intermixed configurations. Since our method for obtaining the layer-resolved shifts cannot account for volume relaxations, a fixed theoretical lattice constant equal to the surrounding magnetic metal is applied in all multilayer systems. If the influence from interface specific effects is small, it is expected that the dispersion of the ICLSs is largely determined by the local coordination, ${ }^{2}$ following the trend of the disordered alloys in Figs. 2-4 for the fixed metal volume. Here we focus on the general trends over a wide range of systems, considering fully embedded thin films. Details of the respective concentration profiles and layer resolved shifts are listed in the Appendix.

\section{1. $\mathrm{Cu} / \mathrm{Ni}$}

The $\mathrm{Cu} 2 p_{3 / 2}$ interface CLSs in the $\mathrm{Ni} / \mathrm{Cu}_{N} / \mathrm{Ni}$ fcc (100) systems are shown in Fig. 6 for the three different interface qualities and $N=1-10$ MLs thicknesses. The long-dashed lines correspond to the bulk metal $\mathrm{Cu}$ shift, with the volume of the surrounding fcc Ni metal, while the dotted lines denote the dilute $\mathrm{Cu}$ atom limit. The ideal interface $\left(\Gamma_{C}=0\right)$ is shown in the left panels, displaying a rapid and smooth increase of the shifts as a function of the number of $\mathrm{Cu}$ layers. Here, the inner layer $\mathrm{Cu}$ shift (large solid symbols) tends to the positive 

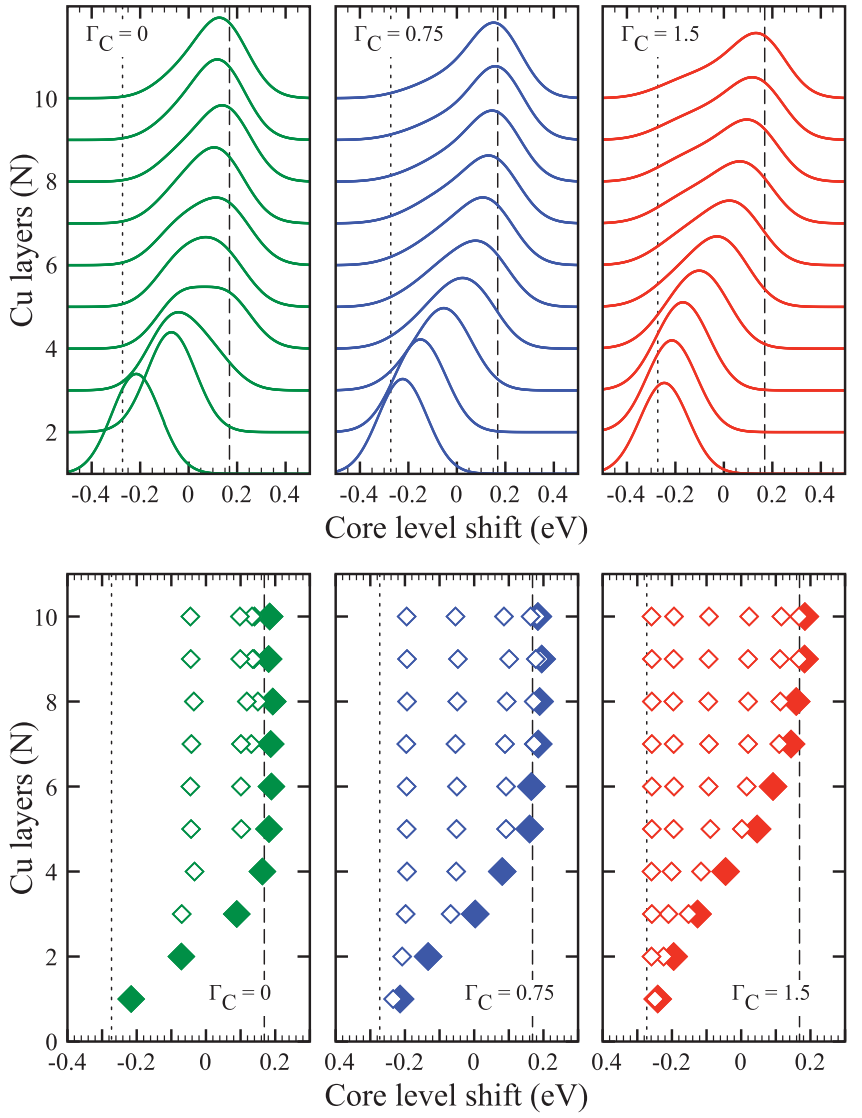

FIG. 6. (Color online) $\mathrm{Cu} 2 p_{3 / 2}$ ICLSs in the $\mathrm{Ni} / \mathrm{Cu}_{N} / \mathrm{Ni}$ fcc (100) system for different values of the interface alloying parameter $\Gamma_{C}=0$ (green), 0.75 (blue), and 1.5 (red). The continuous curves in the upper part are the corresponding broadened spectra according to Eq. (6), while the symbols in the lower part represent core-level shifts of individual atomic layers. In the lower part, the solid symbols represent the CLS of the atoms in the center of the $\mathrm{Cu}$ slab. For comparison, the CLS of bulk $\mathrm{Cu}$ at $\mathrm{Ni}$ metal volume (long-dashed lines) and of $\mathrm{Cu}$ impurities in a dilute $\mathrm{Cu} / \mathrm{Ni}$ alloy (dotted lines) are shown.

pressure induced bulk metal CLS, while the layers closer to interfaces (empty symbols) are smaller or negative. The largest difference between the inner and outer layers is $0.22 \mathrm{eV}$. A characteristic CLS is found for the sharp interface already at the two $\mathrm{Cu}$ layer system. In the middle panels in Fig. 6, the perfect interface is destroyed by applying the interface alloying, $\Gamma_{C}=0.75$. The result is that the increase of the shift as a function of $\mathrm{Cu}$ MLs is not as fast. The outer layers are now represented by mixed alloys and give more negative shifts, closer to the dilute limit. This can be compared to Fig. 2, with the more negative CLS for higher Ni concentration. The total range of the shifts has also increased to $0.4 \mathrm{eV}$. For the further increase of interface alloying $\left(\Gamma_{C}=1.50\right)$ in the right panel, the inner layer shifts increase more slowly as a function of spacer thickness. The spread of the CLS is now doubled compared to the case of the perfect interfaces. It is interesting to note that the bulk metal value as well as the dilute limit of the layered systems correspond well with the shifts obtained at the fixed volume for the disordered CuNi alloys; see Fig. 2.
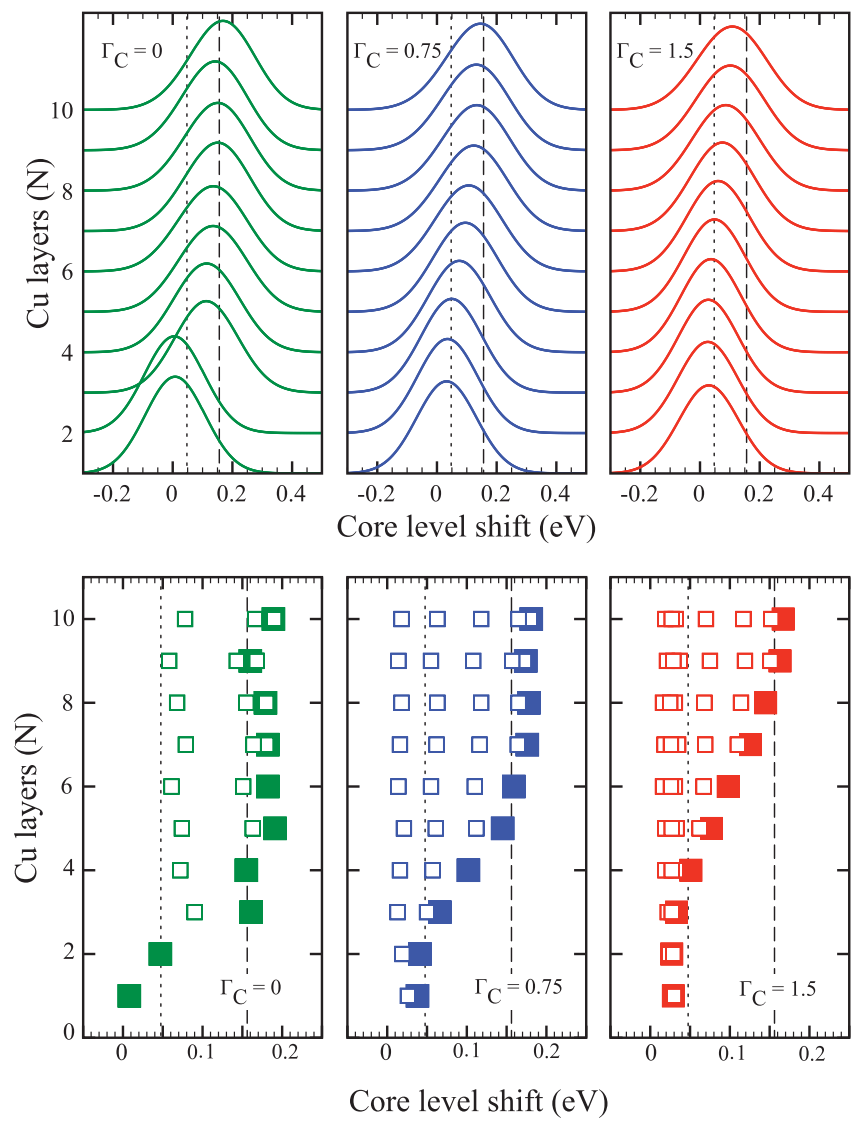

FIG. 7. (Color online) Layer-resolved $\mathrm{Cu} 2 p_{3 / 2}$ ICLSs in the $\mathrm{Co} / \mathrm{Cu}_{N} / \mathrm{Co} \mathrm{fcc}(100)$ system for different values of the interface alloying parameter $\Gamma_{C}$. The details of the figure are described in the caption to Fig. 6.

\section{2. $\mathrm{Cu} / \mathrm{Co}$}

Our results for the $\mathrm{Cu}$ ICLS in the embedded thin-film $\mathrm{Co} / \mathrm{Cu}_{N} / \mathrm{Co}$ fcc (100) systems are shown in Fig. 7. As before, the more positive shifts correspond to the inner layers (large solid symbols). While the range of the interface shifts is smaller in comparison with $\mathrm{Cu} / \mathrm{Ni}$, the bulk value for the inner layers is still reached much faster when the interface alloying is small. The results can be compared with the trends in the disordered alloy systems, Fig. 3, considering the $\mathrm{Cu}$ shift at the fixed fcc Co metal volume, $a=3.49 \AA$. Here we note that the corresponding ICLS at the dilute $\mathrm{Cu}$ atom limit is larger than the shift at some intermediate alloy concentrations. This can also be seen for the layered systems with intermixing, middle, and right panels in Fig. 7, with some of the shifts smaller than that of the dilute limit. A noteworthy feature of the ICLS for thin layer thickness and ideal interfaces, which also differs from the disordered alloy results, is the "jump" between 2 and $3 \mathrm{MLs} \mathrm{Cu}$. This feature could be due to a weak effect from quantum well states that are known to be present in the system. $^{49}$

\section{3. $\mathrm{Cu} / \mathrm{Fe}$}

Finally, in Fig. 8 we display the calculated $\mathrm{Cu}$ interface shifts in the $\mathrm{Fe} / \mathrm{Cu}_{N} / \mathrm{Fe}$ bcc (100) systems. It is clearly seen that the results differ markedly from the $\mathrm{Cu} / \mathrm{Ni}$ and $\mathrm{Cu} / \mathrm{Co}$ cases 

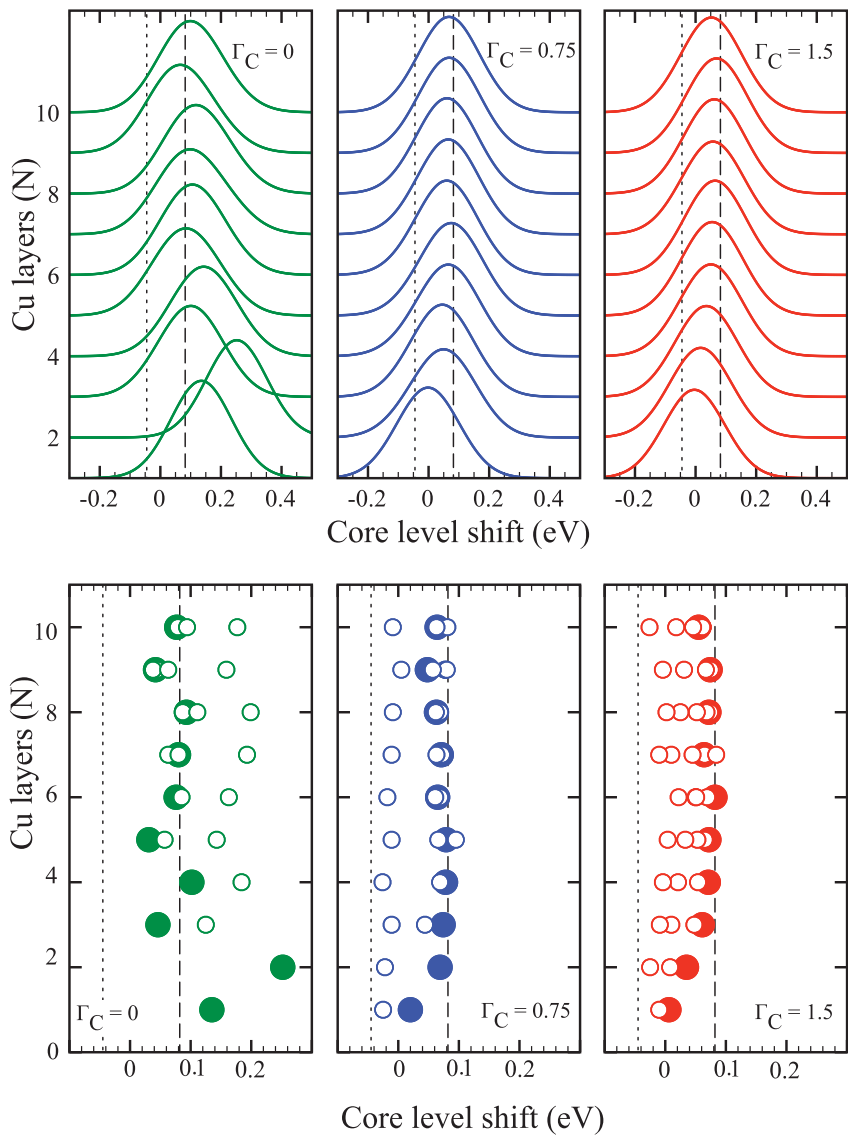

FIG. 8. (Color online) $\mathrm{Cu} 2 p_{3 / 2}$ ICLSs in the $\mathrm{Fe} / \mathrm{Cu}_{N} / \mathrm{Fe}$ fcc (100) system for different values of the interface alloying parameter $\Gamma_{C}$. The details of the figure are described in the caption to Fig. 6 .

when the interfaces are sharp. The shifts do not depend on the layer thickness and are oscillating, though a larger shift is seen for the 2-ML system. If the ideal interfaces are destroyed (i.e., $\left.\Gamma_{C}>0\right)$ the effect disappears. In addition, the behavior differs in comparison with the corresponding disordered alloys, Fig. 4. These facts are strong indications on that the effect is related to the $\mathrm{Fe} / \mathrm{Cu}$ interfaces. In fact, the effect can be attributed to a well-known minority spin interface state at the $\mathrm{Fe} / \mathrm{Cu}$ bcc (100) interface. ${ }^{50}$

In Fig. 9 we show the layer and $k_{\|}$resolved minority spin spectral function of an $\mathrm{Fe} / \mathrm{Cu}$ interface. Only atomic layers at the interface and one layer away are shown. In the left panel there is no alloying of the interface, i.e., $\Gamma_{C}=0$ and in the right panel the interface roughness is slightly alloyed with the parameter $\Gamma_{C}=0.6$. The spectral function has been increased by a factor of 10 for the $\mathrm{Cu}$ layers in order to be visible. It can be seen in the left panel that there is a feature located between the $\bar{\Gamma}$ and the $\bar{X}$ points in the surface Brillouin zone and close to the Fermi energy at the interface. This feature has been investigated before and is attributed to an interface state. ${ }^{50}$ When the interface is disordered this feature changes very rapidly and for the alloying parameter $\Gamma_{C}>0.60$ it has almost disappeared. The larger shift for $N=2$ occurs possibly due to the interaction between interface states, which could be turned on and off by switching the magnetic alignment, i.e., from ferromagnetic to antiferromagnetic; see Ref. 50.
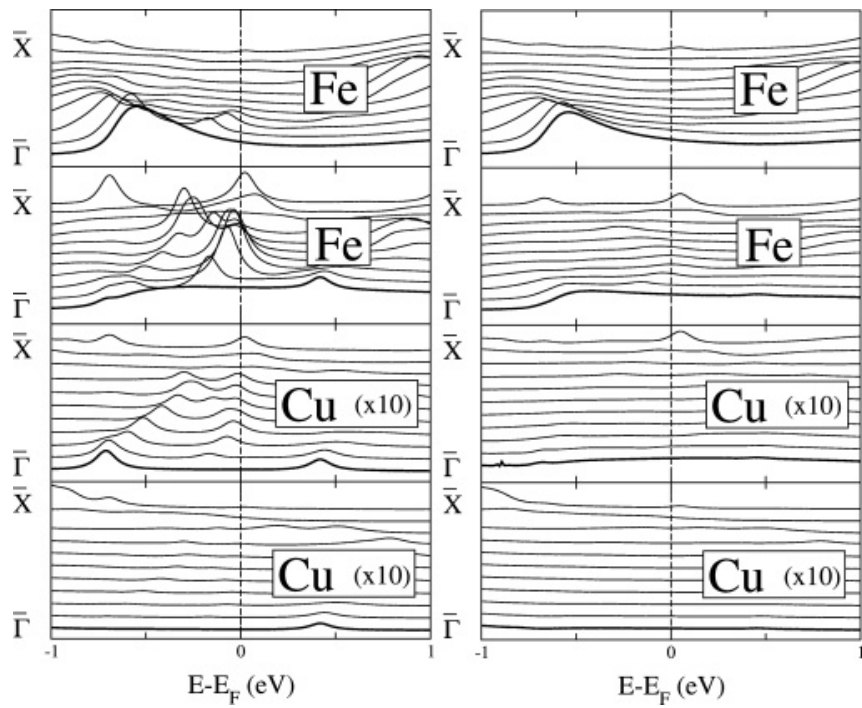

FIG. 9. Spectral density along the $\bar{\Gamma}-\bar{X}$ direction in the twodimensional Brillouin zone (observe that the point $\bar{\Gamma}$ has nothing to do with interface quality). The thick lines correspond to the $\bar{\Gamma}$ point. The left panel is for ideal interfaces, i.e., when the interface roughness is zero. The right panel is for interface roughness $\Gamma_{C}=0.60$. Note how the interface states have almost vanished on the right side.

\section{CONCLUSIONS}

We have demonstrated the use of theoretical layer-resolved core-level shifts as a way to compare different interface qualities, using only a single parameter to describe the atom intermixing profiles in different thin-film nanosystems. This allows for conceptually simple and efficient calculations. We have presented detailed layer-resolved core-level shifts for $\mathrm{Cu}$ $2 p_{3 / 2}$ in combination with $\mathrm{Ni}, \mathrm{Fe}$, and $\mathrm{Co}$ that may serve as a basis for modeling more complicated interface structures on the subnanometer scale.

Investigations were performed for fcc $\mathrm{Ni} / \mathrm{Cu}$, fcc $\mathrm{Co} / \mathrm{Cu}$, and bec $\mathrm{Fe} / \mathrm{Cu}$ sandwiches, which displayed different behaviors of the shifts as a function of interface quality, i.e., atom intermixing, and for the total number of spacer atoms in the systems. While the $\mathrm{Cu}-\mathrm{Ni}$ systems showed smooth trends for the shifts over $N$ total layers, more abrupt tendencies were found in the Co and Fe materials, with a profoundly different behavior in the $\mathrm{Cu}-\mathrm{Fe}$ system attributed to special interface states in Fe. This clearly demonstrates a way for distinguishing between ideal and more intermixed interfaces, as well as the possibility of detecting properties that originate from interface interference effects.

\section{ACKNOWLEDGMENTS}

Financial support from the Swedish Research Council (VR), the LiLi-NFM Center and the Swedish e-Science Research Centre (SeRC), and the Göran Gustafsson Foundation for Research in Natural Sciences and Medicine are acknowledged. E.H. acknowledges financial support from FONDECYT Grant No. 1110602, Chile. Dr. Nicolas Bock is also acknowledged for fruitful discussions. 


\section{APPENDIX}

TABLE II. The interface core-level shifts of multilayers with intermixing parameter $\Gamma=0.00$.

\begin{tabular}{|c|c|c|c|c|c|}
\hline $\mathrm{N}$ & $\mathrm{L}$ & Conc. & $\mathrm{Cu} / \mathrm{Ni}$ & $\mathrm{Cu} / \mathrm{Co}$ & $\mathrm{Cu} / \mathrm{Fe}$ \\
\hline 1 & 1 & 1.00 & -0.22 & 0.01 & 0.14 \\
\hline 2 & 1 & 1.00 & -0.07 & 0.04 & 0.25 \\
\hline 3 & 1 & 1.00 & 0.09 & 0.16 & 0.05 \\
\hline 3 & 2 & 1.00 & -0.07 & 0.09 & 0.13 \\
\hline 4 & 1 & 1.00 & 0.16 & 0.16 & 0.10 \\
\hline 4 & 2 & 1.00 & -0.03 & 0.07 & 0.18 \\
\hline 5 & 1 & 1.00 & 0.18 & 0.19 & 0.03 \\
\hline 5 & 2 & 1.00 & 0.10 & 0.16 & 0.06 \\
\hline 5 & 3 & 1.00 & -0.04 & 0.07 & 0.14 \\
\hline 6 & 1 & 1.00 & 0.19 & 0.18 & 0.08 \\
\hline 6 & 2 & 1.00 & 0.10 & 0.15 & 0.09 \\
\hline 6 & 3 & 1.00 & -0.05 & 0.06 & 0.16 \\
\hline 7 & 1 & 1.00 & 0.19 & 0.18 & 0.08 \\
\hline 7 & 2 & 1.00 & 0.13 & 0.18 & 0.06 \\
\hline 7 & 3 & 1.00 & 0.10 & 0.16 & 0.08 \\
\hline 7 & 4 & 1.00 & -0.04 & 0.08 & 0.19 \\
\hline 8 & 1 & 1.00 & 0.19 & 0.18 & 0.09 \\
\hline 8 & 2 & 1.00 & 0.15 & 0.18 & 0.09 \\
\hline 8 & 3 & 1.00 & 0.12 & 0.16 & 0.11 \\
\hline 8 & 4 & 1.00 & -0.04 & 0.07 & 0.20 \\
\hline 9 & 1 & 1.00 & 0.18 & 0.16 & 0.04 \\
\hline 9 & 2 & 1.00 & 0.14 & 0.17 & 0.05 \\
\hline 9 & 3 & 1.00 & 0.14 & 0.17 & 0.04 \\
\hline 9 & 4 & 1.00 & 0.10 & 0.14 & 0.06 \\
\hline 9 & 5 & 1.00 & -0.04 & 0.06 & 0.16 \\
\hline 10 & 1 & 1.00 & 0.18 & 0.19 & 0.08 \\
\hline 10 & 2 & 1.00 & 0.14 & 0.19 & 0.09 \\
\hline 10 & 3 & 1.00 & 0.13 & 0.19 & 0.08 \\
\hline 10 & 4 & 1.00 & 0.1 & 0.17 & 0.10 \\
\hline 10 & 5 & 1.00 & -0.05 & 0.08 & 0.18 \\
\hline
\end{tabular}

TABLE III. The interface core-level shifts of multilayers with intermixing parameter $\Gamma=0.75$.

\begin{tabular}{|c|c|c|c|c|c|}
\hline $\mathrm{N}$ & $\mathrm{L}$ & Conc. & $\mathrm{Cu} / \mathrm{Ni}$ & $\mathrm{Cu} / \mathrm{Co}$ & $\mathrm{Cu} / \mathrm{Fe}$ \\
\hline 1 & 1 & 0.50 & -0.21 & 0.04 & 0.02 \\
\hline 1 & 2 & 0.23 & -0.23 & 0.03 & -0.03 \\
\hline 1 & 3 & 0.02 & - & - & - \\
\hline 2 & 1 & 0.72 & -0.13 & 0.04 & 0.07 \\
\hline 2 & 2 & 0.25 & -0.21 & 0.02 & -0.02 \\
\hline 2 & 3 & 0.02 & - & - & - \\
\hline 3 & 1 & 0.95 & 0.00 & 0.07 & 0.07 \\
\hline 3 & 2 & 0.75 & -0.07 & 0.05 & 0.04 \\
\hline 3 & 3 & 0.25 & -0.20 & 0.01 & -0.01 \\
\hline 3 & 4 & 0.02 & - & - & - \\
\hline 4 & 1 & 0.98 & 0.09 & 0.10 & 0.08 \\
\hline 4 & 2 & 0.75 & -0.05 & 0.06 & 0.07 \\
\hline 4 & 3 & 025 & -0.20 & 0.02 & -0.03 \\
\hline 4 & 4 & 0.02 & - & - & - \\
\hline 5 & 1 & 1.00 & 0.16 & 0.15 & 0.08 \\
\hline 5 & 2 & 0.98 & 0.10 & 0.11 & 0.10 \\
\hline 5 & 3 & 0.75 & -0.06 & 0.06 & 0.07 \\
\hline 5 & 4 & 0.25 & -0.20 & 0.02 & -0.01 \\
\hline 5 & 5 & 0.02 & - & - & - \\
\hline 6 & 1 & 1.00 & 0.17 & 0.16 & 0.07 \\
\hline 6 & 2 & 0.98 & 0.10 & 0.11 & 0.07 \\
\hline 6 & 3 & 0.75 & -0.05 & 0.06 & 0.06 \\
\hline 6 & 4 & 0.25 & -0.20 & 0.01 & -0.02 \\
\hline 6 & 5 & 0.02 & - & - & - \\
\hline 7 & 1 & 1.00 & 0.19 & 0.18 & 0.07 \\
\hline 7 & 2 & 1.00 & 0.18 & 0.16 & 0.07 \\
\hline 7 & 3 & 0.98 & 0.09 & 0.12 & 0.07 \\
\hline 7 & 4 & 0.75 & -0.05 & 0.06 & 0.06 \\
\hline 7 & 5 & 0.25 & -0.20 & 0.02 & -0.01 \\
\hline 7 & 6 & 0.02 & - & - & - \\
\hline 8 & 1 & 1.00 & 0.19 & 0.18 & 0.06 \\
\hline 8 & 2 & 1.00 & 0.18 & 0.17 & 0.06 \\
\hline 8 & 3 & 0.98 & 0.10 & 0.12 & 0.07 \\
\hline 8 & 4 & 0.75 & -0.05 & 0.06 & 0.06 \\
\hline 8 & 5 & 0.25 & -0.20 & 0.02 & -0.01 \\
\hline 8 & 6 & 0.02 & - & - & - \\
\hline 9 & 1 & 1.00 & 0.20 & 0.17 & 0.05 \\
\hline 9 & 2 & 1.00 & 0.19 & 0.17 & 0.08 \\
\hline 9 & 3 & 1.00 & 0.18 & 0.16 & 0.08 \\
\hline 9 & 4 & 0.98 & 0.10 & 0.11 & 0.08 \\
\hline 9 & 5 & 0.75 & -0.05 & 0.06 & 0.06 \\
\hline 9 & 6 & 0.25 & -0.20 & 0.01 & 0.01 \\
\hline 9 & 7 & 0.02 & - & - & - \\
\hline 10 & 1 & 1.00 & 0.18 & 0.18 & 0.16 \\
\hline 10 & 2 & 1.00 & 0.18 & 0.18 & 0.07 \\
\hline 10 & 3 & 1.00 & 0.16 & 0.17 & 0.07 \\
\hline 10 & 4 & 0.98 & 0.09 & 0.12 & 0.08 \\
\hline 10 & 5 & 0.75 & -0.05 & 0.06 & 0.06 \\
\hline 10 & 6 & 0.25 & -0.20 & 0.02 & -0.01 \\
\hline 10 & 7 & 0.02 & - & - & - \\
\hline
\end{tabular}


TABLE IV. The interface core-level shifts of multilayers with intermixing parameter $\Gamma=1.50$.

\begin{tabular}{|c|c|c|c|c|c|}
\hline $\mathrm{N}$ & $\mathrm{L}$ & Conc. & $\mathrm{Cu} / \mathrm{Ni}$ & $\mathrm{Cu} / \mathrm{Co}$ & $\mathrm{Cu} / \mathrm{Fe}$ \\
\hline 1 & 1 & 0.26 & -0.24 & 0.03 & 0.01 \\
\hline 1 & 2 & 0.21 & -0.25 & 0.03 & -0.01 \\
\hline 1 & 3 & 0.11 & -0.27 & 0.05 & -0.02 \\
\hline 1 & 4 & 0.04 & - & - & - \\
\hline 2 & 1 & 0.47 & -0.20 & 0.03 & 0.04 \\
\hline 2 & 2 & 0.32 & -0.22 & 0.02 & 0.02 \\
\hline 2 & 3 & 0.14 & -0.26 & 0.03 & -0.03 \\
\hline 2 & 4 & 0.05 & - & - & - \\
\hline 3 & 1 & 0.68 & -0.13 & 0.03 & 0.06 \\
\hline 3 & 2 & 0.58 & -0.15 & 0.03 & 0.05 \\
\hline 3 & 3 & 0.36 & -0.21 & 0.02 & 0.01 \\
\hline 3 & 4 & 0.16 & -0.26 & 0.03 & -0.01 \\
\hline 3 & 5 & 0.05 & - & - & - \\
\hline 4 & 1 & 0.79 & -0.05 & 0.05 & 0.07 \\
\hline 4 & 2 & 0.62 & -0.12 & 0.03 & 0.05 \\
\hline 4 & 3 & 0.37 & -0.20 & 0.02 & 0.02 \\
\hline 4 & 4 & 0.16 & -0.26 & 0.03 & -0.00 \\
\hline 4 & 5 & 005 & - & - & - \\
\hline 5 & 1 & 0.90 & 0.05 & 0.08 & 0.07 \\
\hline 5 & 2 & 0.83 & 0.00 & 0.06 & 0.07 \\
\hline 5 & 3 & 0.63 & -0.09 & 0.03 & 0.05 \\
\hline 5 & 4 & 0.37 & -0.20 & 0.02 & 0.03 \\
\hline 5 & 5 & 0.16 & -0.26 & 0.03 & 0.00 \\
\hline 5 & 6 & 0.05 & - & - & - \\
\hline 6 & 1 & 0.94 & 0.09 & 0.10 & 0.08 \\
\hline 6 & 2 & 0.84 & 0.02 & 0.07 & 0.07 \\
\hline 6 & 3 & 0.63 & -0.09 & 0.03 & 0.05 \\
\hline 6 & 4 & 0.36 & -0.19 & 0.02 & 0.05 \\
\hline 6 & 5 & 0.16 & -0.26 & 0.03 & 0.02 \\
\hline 6 & 6 & 0.05 & - & - & - \\
\hline 7 & 1 & 0.98 & 0.14 & 0.13 & 0.06 \\
\hline 7 & 2 & 0.95 & 0.11 & 0.11 & 0.07 \\
\hline 7 & 3 & 0.84 & 0.02 & 0.07 & 0.08 \\
\hline 7 & 4 & 0.63 & -0.09 & 0.04 & 0.05 \\
\hline 7 & 5 & 0.37 & -0.19 & 0.02 & 0.01 \\
\hline 7 & 6 & 0.16 & -0.26 & 0.03 & -0.01 \\
\hline 7 & 7 & 0.05 & - & - & - \\
\hline 8 & 1 & 0.99 & 0.16 & 0.15 & 0.07 \\
\hline 8 & 2 & 0.95 & 0.11 & 0.11 & 0.07 \\
\hline 8 & 3 & 0.84 & 0.02 & 0.07 & 0.07 \\
\hline 8 & 4 & 0.63 & -0.10 & 0.03 & 0.05 \\
\hline 8 & 5 & 0.37 & -0.20 & 0.02 & 0.03 \\
\hline 8 & 6 & 0.16 & -0.26 & 0.03 & 0.00 \\
\hline 8 & 7 & 0.05 & - & - & - \\
\hline 9 & 1 & 1.00 & 0.18 & 0.16 & 0.07 \\
\hline 9 & 2 & 0.99 & 0.17 & 0.15 & 0.08 \\
\hline 9 & 3 & 0.95 & 0.11 & 0.12 & 0.08 \\
\hline 9 & 4 & 0.84 & 0.02 & 0.08 & 0.08 \\
\hline 9 & 5 & 0.63 & -0.09 & 0.04 & 0.07 \\
\hline 9 & 6 & 0.37 & -0.20 & 0.02 & 0.03 \\
\hline 9 & 7 & 0.16 & -0.26 & 0.03 & -0.00 \\
\hline 9 & 8 & 0.05 & - & - & - \\
\hline 10 & 1 & 1.00 & 0.18 & 0.17 & 0.06 \\
\hline 10 & 2 & 0.99 & 0.17 & 0.15 & 0.06 \\
\hline 10 & 3 & 0.95 & 0.12 & 0.12 & 0.06 \\
\hline 10 & 4 & 0.84 & 0.02 & 0.07 & 0.06 \\
\hline 10 & 5 & 0.63 & -0.09 & 0.03 & 0.05 \\
\hline 10 & 6 & 0.37 & -0.19 & 0.02 & 0.02 \\
\hline 10 & 7 & 0.16 & -0.26 & 0.03 & -0.03 \\
\hline 10 & 8 & 0.05 & - & - & - \\
\hline
\end{tabular}


${ }^{1}$ B. Johansson and N. Mårtensson, Phys. Rev. B 21, 4427 (1980).

${ }^{2}$ W. Olovsson, E. Holmström, J. Wills, P. James, I. A. Abrikosov, and A. M. N. Niklasson, Phys. Rev. B 72, 155419 (2005).

${ }^{3}$ I. A. Abrikosov, W. Olovsson, and B. Johansson, Phys. Rev. Lett. 87, 176403 (2001).

${ }^{4}$ E. Holmström et al., Phys. Rev. Lett. 97, 266106 (2006).

${ }^{5}$ E. Holmström, L. Nordström, L. Bergqvist, B. Skubic, B. Hjörvarsson, I. A. Abrikosov, P. Svedlindh, and O. Eriksson, Proc. Natl. Acad. Sci. USA 101, 4742 (2004).

${ }^{6}$ P. Soven, Phys. Rev. 156, 809 (1967).

${ }^{7}$ B. Velický, S. Kirkpatrick, and H. Ehrenreich, Phys. Rev. 175, 747 (1968).

${ }^{8}$ H. L. Skriver and N. M. Rosengaard, Phys. Rev. B 43, 9538 (1991).

${ }^{9}$ I. A. Abrikosov and H. L. Skriver, Phys. Rev. B 47, 16532 (1993).

${ }^{10}$ A. V. Ruban and H. L. Skriver, Comput. Mater. Sci 15, 119 (1999).

${ }^{11}$ P. Hohenberg and W. Kohn, Phys. Rev. 136, B864 (1964).

${ }^{12}$ W. Kohn and L. Sham, Phys. Rev. 140, A1133 (1965).

${ }^{13}$ S. Hüfner and G. K. Wertheim, Phys. Lett. A 51, 299 (1975).

${ }^{14}$ W. Olovsson, C. Göransson, L. V. Pourovskii, B. Johansson, and I. A. Abrikosov, Phys. Rev. B 72, 064203 (2005).

${ }^{15}$ T. Marten, W. Olovsson, S. I. Simak, and I. A. Abrikosov, Phys. Rev. B 72, 054210 (2005).

${ }^{16}$ S. Granroth et al., Phys. Rev. B 80, 094104 (2009).

${ }^{17}$ W. Olovsson, E. Holmström, T. Marten, B. Johansson, and I. A. Abrikosov, J. Electron Spectrosc. Relat. Phenom. 178-9, 88 (2010).

${ }^{18}$ S. Granroth, W. Olovsson, E. Holmström, R. Knut, M. Gorgoi, S. Svensson, and O. Karis, J. Electron Spectrosc. Relat. Phenom. 183, 80 (2011).

${ }^{19}$ O. K. Andersen, Phys. Rev. B 12, 3060 (1975).

${ }^{20}$ H. L. Skriver, The LMTO Method (Springer-Verlag, Berlin, 1984).

${ }^{21}$ O. K. Andersen and O. Jepsen, Phys. Rev. Lett. 53, 2571 (1984).

${ }^{22}$ O. K. Andersen, O. Jepsen, and D. Glötzel, Highlights of Condensed-Matter Theory edited by F. Bassani, F. Fumi and M. P. Tosi (North-Holland, New York, 1985).

${ }^{23}$ I. Turek, V. Drchal, J. Kudrnovský, M. S̆ob, and P. Weinberger, Electronic Structure of Disordered Alloys, Surfaces and Interfaces (Kluwer, Norwell, MA, 1997).

${ }^{24}$ B. Wenzien, J. Kudrnovský, V. Drchal, and M. Sob, J. Phys. Condens. Matter 1, 9893 (1989).

${ }^{25}$ B. Velický and J. Kudrnovský, Surf. Sci. 21, 93 (1977).

${ }^{26}$ J. Kudrnovský, P. Weinberger, and V. Drchal, Phys. Rev. B 44, 6410 (1991).

${ }^{27}$ I. A. Abrikosov, A. M. N. Niklasson, S. I. Simak, B. Johansson, A. V. Ruban, and H. L. Skriver, Phys. Rev. Lett. 76, 4203 (1996).

${ }^{28}$ I. A. Abrikosov, S. I. Simak, B. Johansson, A. V. Ruban, and H. L. Skriver, Phys. Rev. B 56, 9319 (1997).
${ }^{29}$ W. Olovsson, I. A. Abrikosov, and B. Johansson, J. Electron Spectrosc. Relat. Phenom. 127, 65 (2002).

${ }^{30}$ M. Aldén, H. L. Skriver, and B. Johansson, Phys. Rev. Lett. 71, 2449 (1993).

${ }^{31}$ M. Aldén, I. A. Abrikosov, B. Johansson, N. M. Rosengaard, and H. L. Skriver, Phys. Rev. B 50, 5131 (1994).

${ }^{32}$ W. Olovsson, C. Göransson, T. Marten, and I. A. Abrikosov, Phys. Status Solidi B 243, 2447 (2006).

${ }^{33}$ A. V. Ruban and H. L. Skriver, Phys. Rev. B 55, 8801 (1997).

${ }^{34}$ A. Zunger, S.-H. Wei, L. G. Ferreira, and J. E. Bernard, Phys. Rev. Lett. 65, 353 (1990).

${ }^{35}$ A. V. Ruban and I. A. Abrikosov, Rep. Prog. Phys. 71, 046501 (2008).

${ }^{36}$ J. P. Perdew, K. Burke, and M. Ernzerhof, Phys. Rev. Lett. 77, 3865 (1996).

${ }^{37}$ B. Skubic, E. Holmström, D. Iusan, O. Bengone, O. Eriksson, R. Brucas, B. Hjörvarsson, V. Stanciu, and P. Nordblad, Phys. Rev. Lett. 96, 057205 (2006).

${ }^{38}$ B. Skubic, E. Holmström, O. Eriksson, A. M. Blixt, G. Andersson, B. Hjörvarsson, and V. Stanciu, Phys. Rev. B 70, 094421 (2004).

${ }^{39}$ G. Andersson, A. M. Blixt, V. Stanciu, B. Skubic, E. Holmström, and P. Nordblad, J. Magn. Magn. Mater. 267, 234 (2003).

${ }^{40}$ B. Skubic, E. Holmström, A. Bergman, and O. Eriksson, Phys. Rev. B 77, 144408 (2008).

${ }^{41}$ M. Parnaste, M. Marcellini, E. Holmström, N. Bock, J. Fransson, O. Eriksson, and B. Hjörvarsson, J. Phys. Condens. Matter 19, 246213 (2007).

${ }^{42}$ A. M. Blixt, G. Andersson, V. Stanciu, B. Skubic, E. Holmström, P. Nordblad, and B. Hjörvarsson, J. Magn. Magn. Mater. 280, 346 (2004).

${ }^{43}$ A. M. N. Niklasson, I. A. Abrikosov, and B. Johansson, Phys. Rev. B 58, 3613 (1998).

${ }^{44}$ P. Steiner and S. Hüfner, Acta Metall. 29, 1885 (1981).

${ }^{45}$ P. F. Barbieri, A. de Siervo, M. F. Carazolle, R. Landers, and G. G. Kleiman, J. Electron Spectrosc. Relat. Phenom. 135, 113 (2004).

${ }^{46}$ M. Ushida, K. Tanaka, K. Sumiyama, and Y. Nakamura, J. Phys. Soc. Jpn. 58, 1725 (1989).

${ }^{47}$ E. Santos Jr., M. Abbate, T. A. Grandi, and J. C. de Lima, J. Alloys Compd. 346, 24 (2002).

${ }^{48}$ T. Marten, I. A. Abrikosov, W. Olovsson, B. Johansson, R. J. Cole, G. Beamson, S. R. Haines, and P. Weightman, Phys. Rev. B 79, 012201 (2009).

${ }^{49}$ J. E. Ortega and F. J. Himpsel, Phys. Rev. Lett. 69, 844 (1992).

${ }^{50}$ A. M. N. Niklasson, L. Nordström, S. Mirbt, B. Johansson, and H. L. Skriver, J. Phys. Condens. Matter 11, 975 (1999). 